\title{
LIVSIĈ THEORY FOR COMPACT GROUP EXTENSIONS OF HYPERBOLIC SYSTEMS.
}

\author{
DMITRY DOLGOPYAT
}

Dedicated to Yu. S. Ilyashenko on the occasion of his 60th birthday.

\begin{abstract}
We prove Livsiĉ type results for rapidly mixing compact group extensions of Anosov diffeomorphisms.
\end{abstract}

\section{INTRODUCTION.}

Recently several new phenomena in dynamics were discovered by looking at small perturbations of compact group extensions of hyperbolic systems $[8,14]$. In view of this it is desirable to develop a general theory of perturbations of such systems. The first step towards this goal is to understand infinitesimal perturbations that is to study homological equations over such systems. In this note we study regularity of solutions to cocycle equations. Regularity theory plays an important role in rigidity theory. Two of the most studied cases are translations of $\mathbb{T}^{d}$ and Anosov diffeomorphisms (see [7, 11, 15] for the analysis of some other systems). The systems considered in our paper exhibit a mixture of hyperbolic and elliptic behaviors.

Let $M$ be a compact $C^{\infty}$ Riemannian manifold and $f: M \rightarrow M$ be an Anosov diffeomorphism. Let $G$ be a compact connected Lie group, $H$ a Lie subgroup of $G, Y=G / H$ and $\tau \in C^{\infty}(M, G)$. Let $N=M \times Y$. Define $F: N \rightarrow N$ by $F(x, y)=(f x, \tau(x) y)$. We say that a function $A$ on $N$ is a coboundary if

$$
A=B-B \circ F .
$$

If $B$ is bounded, Hölder, smooth etc. we say that $A$ is a bounded, Hölder, smooth etc. coboundary. Let $\phi \in C^{\alpha}(M), \mu_{\phi}$ be the Gibbs state with potential $\phi$ and $d \nu_{\phi}=d \mu_{\phi} d y$.

In this note we prove the following. Fix $z_{0} \in N$.

Theorem 1. Let $F$ be rapidly mixing. Let $A \in C^{\infty}(N)$ be a coboundary in $L^{2}\left(\nu_{\phi}\right)$ for some Hölderfunction $\phi$. Then $A$ is $C^{\infty}$ coboundary. In

2000 Mathematics Subject Classification. 3730C, 37D30, 37J40.

Key words and phrases. cocycle equation, transfer operator, partial hyperbolicity, small divisors. 
particular, if $A$ is a bounded coboundary then it is a $C^{\infty}$ coboundary. Moreover there exists $k_{0}$ such that if $A$ belongs to $C^{k}(N)$ then $B$ belongs to $C^{k-k_{0}}(N)$. If $B$ satisfies a normalization condition

$$
B\left(z_{0}\right)=0
$$

then

$$
\|B\|_{k-k_{0}} \leq \text { Const }\|A\|_{k}
$$

We refer the reader to the next section for the definition of rapid mixing. Recall ([4]) that it holds for generic extension.

Observe that Theorem 1 implies in particular that the set of coboundaries is closed in $C^{k}$ for $k>k_{0}$.

We also present versions of this theorem for extensions of subshifts of finite type.

Our results are also true for relative coboundaries. Let $A_{0}(x)=$ $\int A(x, y) d y$. We say that $A$ is a relative coboundary if

$$
A=A_{0}+B-B \circ F \text {. }
$$

Theorem 2. The results of Theorem 1 are valid also for relative coboundaries.

\section{Preliminaries.}

2.1. Subshifts of finite type. Here we present some results about subshifts of finite type and their compact extensions. The proofs can be found in [13], Chapters 3 and 8. For a geometric interpretation of the results about the extensions see e.g. [4], Section 2. Let $\mathfrak{a}$ be a finite alphabet, $\mathbf{A}$ be $\operatorname{Card}(\mathfrak{a}) \times \operatorname{Card}(\mathfrak{a})$-matrix whose entries are zeroes and ones. Let $\Sigma=\Sigma_{\mathbf{A}}$ be associated (two-sided) subshift of finite type, that is $\Sigma=\left\{\left\{\omega_{i}\right\}_{i=-\infty}^{+\infty}\right.$ such that $\omega_{i} \in \mathfrak{a}$ and $\left.\mathbf{A}_{\omega_{i}, \omega_{i+1}}=1\right\}$. Let $\sigma$ be a shift $\sigma(\omega)_{i}=\omega_{i+1}$. Given $\theta<1$ we consider the metric $d_{\theta}$ on $\Sigma$ given by $d_{\theta}\left(\omega^{\prime}, \omega^{\prime \prime}\right)=\theta^{j}$ where $j=\max \left(k: \omega_{i}^{\prime}=\omega_{i}^{\prime \prime}\right.$ for $\left.|i|<k\right)$. Let $C_{\theta}(\Sigma)$ denote the set of $d_{\theta}$-Lipschitz functions. Given $\phi \in C_{\theta}(\Sigma)$ we denote by $\mu_{\phi}$ the Gibbs measure with potential $\phi$, that is

$$
h_{\mu_{\phi}}+\mu_{\phi}(\phi)=\sup _{\mu}\left(h_{\mu}+\mu(\phi)\right)
$$

where the supremum is taken over all $\sigma$ invariant probability measures. We will use the fact that homologous functions have the same Gibbs measures. Let $G$ be a compact connected Lie group and $\tau \in C_{\theta}(\Sigma, G)$. Let $\tilde{N}=\Sigma \times Y$. Define $\tilde{F}: \tilde{N} \rightarrow \tilde{N}$ by $F(\omega, y)=(\sigma \omega, \tau(x) y)$. Consider 
a measure $\nu_{\phi}$ given by $d \nu_{\phi}=d \mu_{\phi} d g$. Let $C_{k, \theta}(\tilde{N})=C_{\theta}\left(\Sigma, C^{k}(G)\right)$. We say that $\tilde{F}$ is rapidly mixing if $\forall \phi \forall N \exists k$ such that $\forall A, B \in C_{k, \theta}$

$$
\left|\nu_{\phi}\left(A(\omega, y) B\left(\tilde{F}^{n}(\omega, y)\right)\right)-\nu_{\phi}(A) \nu_{\phi}(B)\right| \leq \mathrm{Const}\|A\|_{k, \theta}\|B\|_{k, \theta} n^{-N} .
$$

It is shown in ([4], Theorem 4.3) that rapid mixing is generic among compact group extensions of subshifts of finite type.

Let $\Sigma^{+}$be associated one-sided subshift which is defined similarly to $\Sigma$ but omega is now an one-sided sequence $\omega=\left\{\omega_{i}\right\}_{i=0}^{\infty} . C_{\theta}\left(\Sigma^{+}\right)$, Gibbs states, rapid mixing etc. are defined for one-sided shifts similarly to two-sided shifts. Let $\tilde{F}: \tilde{N} \rightarrow \tilde{N}$ be a skew extension defined by $\tau \in C_{\theta}(\Sigma, G), \phi \in C_{\theta}(\Sigma)$ be a potential and $A \in C_{k, \theta}$ be an observable. Then there are $\tau^{*} \in C_{\sqrt{\theta}}(\Sigma, G), M \in C_{\sqrt{\theta}}(\Sigma, G), \phi^{*} \in C_{\theta}(\Sigma) \psi \in$ $C_{\sqrt{\theta}}(\Sigma), A^{*} \in C_{k, \sqrt{\theta}}\left(\Sigma^{+}\right), K \in C_{k, \sqrt{\theta}}(\Sigma)$ such that

$$
\tau^{*}=(M \circ \sigma) \tau M^{-1}, \quad \phi^{*}=\phi+\psi-(\psi \circ \sigma), \quad A^{*}=A+K-K \circ \tilde{F} .
$$

Moreover $\phi^{*}$ can be chosen so that

$$
\forall \omega \quad \sum_{\sigma \varpi=\omega} e^{\phi^{*}(\varpi)}=1
$$

Then skew products defined by $\tau$ and $\tau^{*}$ are conjugated, $\phi$ and $\phi^{*}$ have the same Gibbs measure and $A$ is a coboundary iff $A^{*}$ is a coboundary.

Let $\tilde{N}^{+}=\Sigma^{+} \times Y$ and $\tilde{F}$ be a skew extension defined by some $\tau \in C_{\theta}\left(\Sigma^{+}, G\right)$. Let $\Delta$ be a $G$-invariant Laplacian on $G$ and let

$$
H_{\lambda}=\{\varphi: \Delta \varphi=\lambda \varphi\} .
$$

We endow $H_{\lambda}$ with $L^{2}$-norm. Denote $C_{\lambda, \theta}\left(\Sigma^{+}\right)=C_{\theta}\left(\Sigma^{+}, H_{\lambda}\right)$. Let $\phi$ be any Hölderfunction on $\Sigma^{+}$such that

$$
\forall \omega \quad \sum_{\sigma \varpi=\omega} e^{\phi(\varpi)}=1
$$

and let $\mu_{\phi}$ be the Gibbs measure for $\phi$. Consider the transfer operator

$$
(\mathcal{L}(h))(\omega, g)=\sum_{\sigma \varpi=\omega} e^{\phi(\varpi)} h\left(\varpi, \tau^{-1}(\varpi) g\right) .
$$

Then $\mathcal{L}$ preserves $C_{\lambda, \theta}\left(\Sigma^{+}\right)$. Let $\mathcal{L}_{\lambda}$ denote the restriction of $\mathcal{L}$ to $C_{\lambda, \theta}\left(\Sigma^{+}\right)$.

Proposition 1. ([4], Proposition 4.4) If $\tilde{F}$ is rapidly mixing then $\exists C, s$ such that

$$
\left\|\mathcal{L}_{\lambda}^{n}\right\| \leq C \lambda^{s}\left(1-\frac{1}{C \lambda^{s}}\right)^{n}
$$


2.2. Anosov diffeomorphisms. Recall that a diffeomorphism $F$ : $M \rightarrow M$ is called Anosov if there is an $f$-invariant splitting

$$
T M=E^{s} \oplus E^{u}
$$

and constants $C, \rho<1$ such that

$$
\forall v \in E^{s} \quad\left\|d f^{n} v\right\| \leq C \rho^{n}\|v\|, \quad \forall v \in E^{u} \quad\left\|d f^{-n} v\right\| \leq C \rho^{n}\|v\| .
$$

The distributions $E^{s}$ and $E^{u}$ are uniquely integrable, they are tangent to foliation $W^{s}$ and $W^{u}$ respectively. Since $W^{s}$ and $W^{u}$ are transverse, if $x, y \in M$ are close to each other the intersection $W_{l o c}^{u}(x) \cap W_{l o c}^{s}(y)$ consists of one point which we denote by $[x, y]$. A set $\Pi$ is called parallelogram if for all $x, y \in \Pi$ one has $[x, y] \in \Pi$. A partition $\Pi=$ $\left\{\Pi_{1}, \Pi_{2}, \ldots \Pi_{n}\right\}$ is called Markov if for all $x \in \operatorname{Int}\left(\Pi_{i}\right)$

$$
f W_{\Pi}^{s}(x) \in W_{\Pi}^{s}(f x), \quad f^{-1} W_{\Pi}^{u}(x) \in W_{\Pi}^{u}\left(f^{-1} x\right)
$$

where $W_{\Pi}^{*}(z)=W_{l o c}^{*}(z) \bigcap \Pi_{j}$ if $z \in \Pi_{j}$. Given a Markov partition $\Pi$ one can consider a subshift of finite type $\Sigma$ with $\mathfrak{a}=\{1,2 \ldots n\}$ and $\mathbf{A}_{i j}=1$ iff $f\left(\operatorname{Int} P_{i}\right) \bigcap P_{j}=0$. The map $\zeta: \Sigma \rightarrow M$ given by

$$
\zeta(\omega)=\bigcap_{j} f^{-j} \Pi_{\omega_{j}}
$$

defines a semicongugacy between $\sigma$ and $f$. If $\tau$ is a function from $M$ to $G$ let $\bar{\tau}=\tau \zeta$. Then $\zeta \times$ id is a semicongugacy between

$$
F(x, y)=(f x, \tau(x) y) \text { and } \tilde{F}(\omega, y)=(\sigma \omega, \bar{\tau}(\omega) y) .
$$

We shall use the fact that the skew extension $F$ is partially hyperbolic. That is, there is an $F$ invariant splitting

$$
T N=E_{F}^{s} \oplus E_{F}^{c} \oplus E_{F}^{u}
$$

and constants $C, \rho<1$ such that

$$
\forall v \in E_{F}^{s} \quad\left\|d F^{n} v\right\| \leq C \rho^{n}\|v\|, \quad \forall v \in E_{F}^{u} \quad\left\|d F^{-n} v\right\| \leq C \rho^{n}\|v\|
$$

and $E_{F}^{c}$ is the tangent space to the fibers. Gibbs states for $f$ are defined similarly it was done for $\sigma$. An important special case is so called SRB measure which is the Gibbs measure with potential

$$
\phi_{S R B}=-\ln \operatorname{det}\left(d f \mid E^{u}\right) .
$$

The importance of SRB measure comes from the fact that if $\Phi \in C(M)$ then

$$
\frac{1}{n} \sum_{j=0}^{n-1} \Phi\left(f^{j} x\right) \rightarrow \mu_{S R B}(\Phi), \quad n \rightarrow+\infty
$$

for Lebesgue almost all $x$. 
$\forall \alpha \exists \theta$ such that if $\phi \in C^{\alpha}(M)$ then $\bar{\phi}=\phi \circ \zeta \in C_{\theta}(\Sigma)$. Now $\mu_{\phi}$ is a Gibbs state for $f$ iff $\forall \Omega \subset \Sigma$

$$
\mu_{\bar{\phi}}(\Omega)=\mu_{\phi}(\zeta(\Omega)) .
$$

We say that $F$ is rapidly mixing if $\forall \phi \forall N \exists k$ such that $\forall A, B \in C^{k}(M)$

$$
\left|\nu_{\phi}\left(A(x, y) B\left(F^{n}(x, y)\right)\right)-\nu_{\phi}(A) \nu_{\phi}(B)\right| \leq \text { Const }\|A\|_{k}\|B\|_{k} n^{-N} \text {. }
$$

Then $F$ is rapidly mixing iff $\tilde{F}$ is rapidly mixing.

\section{Symbolic SYSTEMS.}

3.1. One-sided shifts. In this subsection let $\tilde{F}: \tilde{N}^{+} \rightarrow \tilde{N}^{+}$be a rapidly mixing extension of one sided subshift. Let $C_{r, \theta}\left(\Sigma^{+}\right)=$ $C_{\theta}\left(\Sigma^{+}, C^{r}(Y)\right)$. We prove the following result.

Lemma 1. Let $A \in C_{\infty, \theta}\left(\Sigma^{+}\right)$be an $L^{2}\left(\nu_{\phi}\right)$ coboundary for some $\phi \in C_{\theta}\left(\Sigma^{+}\right), A=B-B \circ \tilde{F}$, where $B \in L^{2}\left(\nu_{\phi}\right)$. Then $B$ has a version in $C_{\infty, \theta}\left(\Sigma^{+}\right)$. Moreover $\exists k_{0}$ such that if $A \in C_{k, \theta}\left(\Sigma^{+}\right)$, then $B \in C_{k-k_{0}, \theta}\left(\Sigma_{+}\right)$and

$$
\|B\|_{k-k_{0}, \theta} \leq \operatorname{Const}(k)\|A\|_{k, \theta} .
$$

Proof. By the discussion of subsection 2.1 we can assume that $\phi$ satisfies (5). Let $A=A_{0}+\sum_{\lambda \neq 0} A_{\lambda}$, where $A_{0}(\omega)=\int A(\omega, g) d g, A_{\lambda} \in H_{\lambda}$. Let $B=\sum_{\lambda} B_{\lambda}$. Since $F$ commutes with projections to $H_{\lambda}$,

$$
A_{\lambda}=B_{\lambda}-B_{\lambda} \circ \tilde{F} .
$$

In particular, $A_{0}=B_{0}-B_{0} \circ \sigma$ and, by [13], $B \in C_{\theta}\left(\Sigma^{+}\right)$. Hence we can assume without loss of generality that $A_{0} \equiv 0$. Applying $\mathcal{L}_{\lambda}$ to $(8)$ we get

$$
\mathcal{L}_{\lambda} A_{\lambda}=\left(\mathcal{L}_{\lambda}-1\right) B_{\lambda} .
$$

Thus $B_{\lambda}=-\left(1-\mathcal{L}_{\lambda}\right)^{-1} \mathcal{L}_{\lambda} B_{\lambda}$. Now there exists $p=p(G)$ such that

$$
\left\|A_{\lambda}\right\|_{\lambda} \leq \frac{\text { Const }}{\lambda^{k / 2-p}}\|A\|_{k, \theta}
$$

By Proposition 1 there exists $s$ such that

$$
\left\|\left(1-\mathcal{L}_{\lambda}\right)^{-1}\right\| \leq \text { Const } \lambda^{s} .
$$

Hence

$$
\left\|B_{\lambda}\right\|_{\lambda} \leq \operatorname{Const} \lambda^{2 s}\left\|A_{\lambda}\right\|_{\lambda} \leq \frac{\text { Const }}{\lambda^{k / 2-(2 s+p)}}\|A\|_{k, \theta}
$$

Now

$$
\left\|B_{\lambda}\right\|_{k-k_{0}, \theta} \leq \text { Const } \lambda^{\bar{p}+\frac{k-k_{0}}{2}}\left\|B_{\lambda}\right\|_{\lambda} .
$$


Let $B=\sum_{\lambda} B_{\lambda}$. Then

$$
\|B\|_{k-k_{0}, \theta} \leq \sum_{\lambda}\left\|B_{\lambda}\right\|_{k-k_{0}, \theta} \leq \text { Const } \sum_{\lambda} \lambda^{p+\bar{p}+2 s-\left(k_{0} / 2\right)}\|A\|_{k, \theta}
$$

and this series converges if $k_{0}$ is large enough. This completes the proof.

3.2. Two-sided shifts. Let $\tilde{F}: \tilde{N} \rightarrow \tilde{N}$ be an extension of two sided subshift of finite type.

Lemma 2. Let $A=B-B \circ \tilde{F}$. If $A \in C_{\infty, \theta}(\Sigma)$, then $B \in C_{\infty, \theta^{1 / 4}}(\Sigma)$. Moreover $\exists k_{0}$ such that if $A \in C_{k, \theta}(\Sigma)$, then $B \in C_{k-k_{0}, \theta^{1 / 4}}(\Sigma)$ and

$$
\|B\|_{k-k_{0}, \theta^{1 / 4}} \leq \operatorname{Const}(k)\|A\|_{k, \theta} \text {. }
$$

Proof. Let $\tau^{*}=(M \circ \sigma) \tau M^{-1}$. Then the change of variables $y^{*}=M y$ conjugates $\tilde{F}$ and $F^{*}\left(\omega, y^{*}\right)=\left(\sigma \omega, \tau^{*}(\omega) y^{*}\right)$. Thus $A$ is $\tilde{F}$-coboundary iff $A^{*}\left(\omega, y^{*}\right)=A\left(\omega, M^{-1} y^{*}\right)$ is $F^{*}$-coboundary. Write $A^{*}=A^{* *}+K^{*}-$ $K^{*} \circ F^{*}$ where $A^{* *} \in C_{k, \theta^{1 / 4}}\left(\Sigma^{+}\right)$. Then $A^{*}$ is $F^{*}$-coboundary iff $A^{* *}$ is. But by Lemma $1 A^{* *}=B^{* *}-B^{* *} \circ F$ where $B^{* *} \in C_{k-k_{0}, \theta^{1 / 4}}\left(\Sigma^{+}\right)$. Thus

$$
A=(B+K)-(B+k) \circ \tilde{F}
$$

where $B(\omega, y)=B^{* *}(\omega, y), K(\omega, y)=K^{*}(\omega, M y)$. and the statement follows.

Corollary 1. If $\omega$ is a periodic orbit of $\sigma$, say $\sigma^{n} \omega=\omega$, then

$$
\left|\sum_{j=0}^{n-1} A_{\lambda}\left(\tilde{F}^{j}(\omega, y)\right)\right| \leq C \lambda^{s}\left\|A_{\lambda}\right\|_{\theta, k_{0}} d\left(\tau_{n}(\omega) y, y\right) .
$$

Proof.

$\left|\sum_{j=0}^{n-1} A_{\lambda}\left(\tilde{F}^{j}(\omega, y)\right)\right| \leq\left|B_{\lambda}\left(\omega, \tau_{n}(\omega) y\right)-B_{\lambda}(\omega, y)\right| \leq \sqrt{\lambda} d\left(\tau_{n}(\omega) y, y\right)|| B_{\lambda} \|$ and the result follows by (11).

\section{Anosov Diffeomorphisms.}

4.1. Höldercontinuity. Now we start a proof of Theorem 1. In this subsection we show that $B$ has a Hölderversion. Let $\Pi$ be a Markov partition of $M$ and let $\Sigma$ be the associated subshift of a finite type. Let $\zeta: \Sigma \rightarrow M$ be the semiconjugacy

$$
\zeta \circ \sigma=f \circ \zeta .
$$

Let $\bar{\tau}$ and $\tilde{F}$ be as in subsection 2.2. Define $\bar{A}=A \circ \zeta$. Let $\bar{A}=\bar{B}-\bar{B} \circ \tilde{F}$, $\bar{B}=\sum_{\lambda} \bar{B}_{\lambda}$. Let $B_{\lambda}=\bar{B}_{\lambda} \circ \zeta^{-1}, B=\sum_{\lambda} B_{\lambda}$. Since $\zeta^{-1}$ is discontinuous 
we can not deduce immediately that $B_{\lambda}$ are Hölder. Rather we obtain is a consequence of periodic leaves estimates of Corollary 1 . Let $p=(x, y)$ have a dense orbit. We have

$$
B_{\lambda}\left(F^{n} p\right)=B_{\lambda}(p)-\sum_{j=0}^{n-1} A_{\lambda}\left(F^{j} p\right)
$$

Lemma 3. $\left.B_{\lambda}\right|_{\operatorname{Orb}(x, y)}$ is uniformly Höldercontinuous with Hölderconstant $C\left\|A_{\lambda}\right\| \lambda^{s}$.

Proof. Let $m<n$ and

$$
d\left(F^{n} p, F^{m} p\right) \leq \varepsilon .
$$

Denote $k=n-m, z=f^{m} x, q=F^{m} p$. By Anosov Closing Lemma $\exists \tilde{x} \in M$ such that $f^{k} \tilde{x}=\tilde{x}$ and

$$
d\left(f^{j} z, f^{j} y\right) \leq C d\left(f^{k} z, z\right)^{\gamma} \rho^{\max (j, k-j)}
$$

for some $\gamma>0$ and $\rho<1$. Let $u=(\tilde{x}, y)$ then

$$
\left|B_{\lambda}\left(f^{k} q\right)-B_{\lambda}(q)\right|=\left|\sum_{j=0}^{k-1} A_{\lambda}\left(F^{j} q\right)\right| \leq\left|\sum_{j=0}^{k-1} A_{\lambda}\left(F^{j} u\right)\right|+\mid \sum_{j=0}^{k-1}\left[A_{\lambda}\left(F^{j} q\right)-A_{\lambda}\left(\left(F^{j} u\right)\right] \mid .\right.
$$

Now by Corollary 1 the first part is

$$
O\left(\left\|A_{\lambda}\right\| \lambda^{s_{4}} d^{\alpha}\left(p, F^{k} p\right)\right)
$$

and the second part is

$$
O\left(\left\|A_{\lambda}\right\| \sqrt{\lambda} d^{\gamma}\left(p, F^{k} p\right)\right)
$$

since $A_{\lambda}$ is Lipschitz with constant $\sqrt{\lambda}\left\|A_{\lambda}\right\|$.

Since $\operatorname{Orb}(p)$ is dense we can extend $B_{\lambda}$ to Hölderfunctions on $N$.

Lemma 4. Under the conditions of Theorem 1 the restriction of $B$ to each fiber is smooth. Moreover $\exists k_{0}$ such that

$$
\|B\|_{C^{\alpha^{\prime}}\left(M, C^{\left.k-k_{0}(G)\right)} \leq\right.} \leq \operatorname{Const}(k)\|A\|_{C^{\alpha}\left(M, C^{k}(G)\right)} .
$$

Proof. We first show that $B$ has a Hölderversion. By Lemma 3 each $B_{\lambda}$ has an extension from $\operatorname{Orb}(x, g)$ to $N$ which is Hölderwith Höldernorm at most Const $\left\|A_{\lambda}\right\| \lambda^{s}$. By continuity of $A_{\lambda}$ this extension satisfies $A_{\lambda}=$ $B_{\lambda}-B_{\lambda} \circ F$. Now

$$
\left\|A_{\lambda}\right\| \leq \frac{\text { Const }}{\lambda^{(k / 2)-p}}\|A\|_{C^{k}(N)} .
$$

Let $B=\sum_{\lambda} B_{\lambda}$ then

$$
\|B\|_{C^{\alpha}(N)} \leq \sum_{\lambda}\left\|B_{\lambda}\right\|_{C^{\alpha}(N)} \leq \text { Const }\left(\sum_{\lambda} \lambda^{p+s-k / 2}\right)\|A\|_{C^{k}(N)}
$$


and if $k$ is large enough this series converges. In other words there exists $k_{1}$ such that

$$
B_{C^{\alpha}(N)} \leq \text { Const }\|A\|_{C^{k_{1} N}}
$$

Applying this to $\Delta^{m} A$ we get

$$
\begin{gathered}
\|B\|_{C^{\alpha}\left(M, \mathcal{H}^{2 m}(G)\right)} \leq \text { Const }\left\|\Delta^{m} B\right\|_{C^{\alpha}(N)} \leq \text { Const }\left\|\Delta^{m} A\right\|_{C^{k_{1}(N)}} \leq \\
\text { Const }\|A\|_{C^{k_{1}+2 m}(N)}
\end{gathered}
$$

and the result follows by Sobolev embedding theorem.

4.2. Smoothness. We now establish the smoothness of $B$ in the transverse directions.

Lemma 5. Restrictions of $B$ to the leaves of $W_{F}^{s}, W_{F}^{u}$ are smooth.

Proof. It is enough to consider $W_{F}^{s}$. We have $A(p)=B(p)-B(F p)$. Thus $B(p)=A(p)+B(F p)$. Hence if $p \in W^{s}\left(p_{0}\right)$ then

$$
B(p)-B\left(p_{0}\right)=\sum_{j=0}^{\infty}\left[A\left(F^{p} j\right)-A\left(F^{j} p_{0}\right)\right] .
$$

Since $F^{j}$ are contractions on $W_{F}^{S}$ it is clear that this series can be differentiated term by term as many times as we want (see [3] ) .

We now make use of the following fact ([10]).

Proposition 2 (Journe Lemma). Let $\mathcal{F}_{1}$ and $\mathcal{F}_{2}$ be two continuous transverse foliations with smooth leaves. Let $B$ be a continuous function whose restrictions on leaves of $\mathcal{F}_{1}$ and $\mathcal{F}_{2}$ are smooth. Then $B$ is smooth. Moreover there exists $k_{0}$ such that if restrictions of $B$ to the leaves are $C^{k}$ then $B$ is $C^{k-k_{0}}$.

This proposition implies in view of Lemmas 4 and 5 that $B$ is smooth on each leave of $W^{s c}$ and since it is also smooth on each leave of $W^{u}$ we conclude that $B$ is smooth. This complete the proof of Theorem 1 .

Remark. Weaker versions of Journe Lemma proven in [3, 9] would also suffice for the proof.

4.3. Relative coboundaries. Proof of Theorem 2. Apply Theorem 1 to $\Delta A$. 
4.4. A counter-example. Let $G=\mathbb{T}^{2}$,

$$
F\left(x, t_{1}, t_{2}\right)=\left(f x, t_{1}+\alpha_{1} r(x), t_{2}+\alpha_{2} r(x)\right) .
$$

Suppose that $\alpha_{1} / \alpha_{2}$ is irrational and that for all $N$ there exist $m_{1, N}, m_{2, N} \in$ $\mathbb{Z}$ such that

$$
\left|\alpha_{1} m_{1, N}+\alpha_{2} m_{2, N}\right| \leq m_{2, n}^{-N} \text {. }
$$

By reindexing we can assume that $m_{2, N}>N^{2}$. Let $\Phi_{N}\left(x, t_{1}, t_{2}\right)=$ $\exp \left(2 \pi i\left(m_{1, N} t_{1}+m_{2, N} t_{2}\right)\right)$. Then

$$
\Phi \circ F=\exp \left(2 \pi i\left(m_{1, N} \alpha_{1}+m_{2, N} \alpha_{2}\right) r(x)\right) \Phi_{N} .
$$

Let $A=\sum_{N}\left(\left(\Phi_{N}-\Phi \circ F\right) / N^{2}\right)$. Then $A \in C^{\infty}(N), A=B-B \circ F$ where $B=\sum_{N} \Phi_{N} \in C^{0}(N)-C^{1}(N)$. By considering suitable linear combinations of $\Phi_{N}$ it is easy to see that $F$ is not rapidly mixing. This shows that arbitrary extensions need not satisfy Theorem 1.

4.5. Obstructions. We now provide some criteria for function being a coboundary. Most of these criteria come from other papers, however their applicability is a consequence of the fact that different notions of coboundaries coincide in our situation. Sometimes it is easier to verify that $A$ is a relative coboundary. It is also perfectly satisfactory since it is well known when $A_{0}$ is an $f$-coboundary.

(i) Define

$$
\mathcal{D}_{\phi}(A)=\nu_{\phi}\left(A^{2}\right)-\nu_{\phi}^{2}(A)+2 \sum_{j=1}^{\infty}\left[\nu_{\phi}\left(A\left(A \circ \tilde{F}^{j}\right)\right) \nu_{\phi}^{2}(A)\right] .
$$

Proposition 3. ([6]) $A$ is a cohomologous to a constant $\Leftrightarrow \exists \phi$ such that $\mathcal{D}_{\phi}(A)=0 \Leftrightarrow \forall \psi \mathcal{D}_{\phi}(A)=0$.

Proof. Without loss of generality we can assume that $\nu_{\phi}(A)=0$. Then $D_{\phi}(A)=0 \Leftrightarrow A$ is $L^{2}\left(\nu_{\phi}\right)$-coboundary (Spectral theorem)

$\Leftrightarrow A$ is Höldercoboundary (Theorem 1 )

$\Rightarrow \forall \psi \quad A$ is $L^{2}\left(\nu_{\psi}\right)$-coboundary.

(ii) Let $P=\left\{p_{0}, p_{1} \ldots p_{n}\right\}$ be a chain such that $p_{k+1} \in W^{s}\left(p_{k}\right) \cup W^{u}\left(p_{k}\right)$.

We say that $P$ is closed if $p_{0}=p_{n}$. Define

$$
r(P)=\sum_{k} P\left(p_{k}, p_{k+1}\right)
$$

where

$$
r\left(p_{k}, p_{k+1}\right)= \begin{cases}\sum_{j=0}^{\infty}\left[A\left(F^{j} p_{k+1}\right)-A\left(F^{j} p_{k}\right)\right] & \text { if } p_{k+1} \in W^{s} p_{k} \\ \sum_{j=-\infty}^{-1}\left[A\left(F^{j} p_{k}\right)-A\left(F^{j} p_{k+1}\right)\right] & \text { if } p_{k+1} \in W^{s} p_{k}\end{cases}
$$

The following statement is Corollary 3.1 from [11]. 
Proposition 4. If $F$ has accessibility property then $A$ is cohomologous to a constant if and only if for any closed chain $P, r(P)=0$.

(iii) The next result is clear from the proof of Theorem 1.

Proposition 5. $A$ is a relative coboundary $A \Leftrightarrow \forall N \in \mathbb{N} \Delta^{N} A$ is a coboundary.

(iv) Let $G=\mathbb{T}$. Let $\nu$ be the SRB measure for $F$. Consider a one parameter family

$$
F_{\varepsilon}(x, z)=\left(f x, z+\tau(x)+\varepsilon A(x, z)+\varepsilon^{2} \alpha(\varepsilon, x, z) .\right.
$$

Let $\nu_{\varepsilon}$ be any u-Gibbs measure for $F_{\varepsilon}$, that is, the projection of $\nu_{\varepsilon}$ to $M$ is $\mu_{S R B}$.

Proposition 6. $A$ is a relative coboundary if and only if

$$
\lambda_{c}\left(\nu_{\varepsilon}\right)=o\left(\varepsilon^{2}\right) .
$$

Proof. We use the following asymptotics ([5])

$$
\nu_{\varepsilon}(H)=\nu(H)+\varepsilon \omega(H)+o(\varepsilon)
$$

where

$$
\omega(H)=\sum_{j=1}^{\infty} \nu\left(A \circ F^{-j} \frac{d H}{d z}\right) .
$$

We want to apply this to $H_{\varepsilon}=\ln \frac{d F_{\varepsilon}}{d z}$. We have

$$
\frac{d F_{\varepsilon}}{d z}=1+\varepsilon \frac{d A}{d z}+\varepsilon^{2} \frac{d \alpha(0, x, z)}{d z}+o\left(\varepsilon^{2}\right) .
$$

So

$$
\ln \frac{d F_{\varepsilon}}{d z}=\varepsilon \frac{d A}{d z}+\varepsilon^{2}\left[\frac{d \alpha(0, x, z)}{d z}-\frac{1}{2}\left(\frac{d A}{d z}\right)^{2}\right]+o\left(\varepsilon^{2}\right) .
$$

Hence

$$
\begin{gathered}
\nu_{\varepsilon}\left(\ln \frac{d F_{\varepsilon}}{d z}\right)= \\
\varepsilon \nu\left(\frac{d A}{d z}\right)+\varepsilon^{2}\left[\nu\left(\frac{d \alpha(0, x, z)}{d z}\right)-\frac{1}{2} \nu\left(\left(\frac{d A}{d z}\right)^{2}\right)+\sum_{j=1}^{\infty} \nu\left(A \circ F^{-j} \frac{d^{2} A}{d z^{2}}\right)\right]+o\left(\varepsilon^{2}\right) .
\end{gathered}
$$

Now since $d \nu=d \mu_{S R B} d g$ it follows that

$$
\begin{gathered}
\nu\left(\frac{d A}{d z}\right)=\nu\left(\frac{d \alpha(0, x, z)}{d z}\right)=0 \quad \text { and } \\
\nu\left(A \circ f^{-j} \frac{d^{2} A}{d z^{2}}\right)=-\nu\left(\left(\frac{d A}{d z}\right) \circ F^{-j} \frac{d A}{d z}\right) .
\end{gathered}
$$


Hence

$$
\nu_{\varepsilon}\left(\ln \frac{d F_{\varepsilon}}{d z}\right) \sim-\varepsilon^{2}\left[\frac{1}{2} \nu\left(\left(\frac{d A}{d z}\right)^{2}\right)-\sum_{j=1}^{\infty} \nu\left(\left(\frac{d A}{d z}\right) \circ F^{-j} \frac{d A}{d z}\right)\right]
$$

That is

$$
\nu_{\varepsilon}\left(\ln \frac{d F_{\varepsilon}}{d z}\right) \sim-\frac{\varepsilon^{2} \mathcal{D}_{S R B}\left(\frac{d A}{d z}\right)}{2}
$$

Therefore

$$
\lambda_{c}\left(\nu_{\varepsilon}\right)=o\left(\varepsilon^{2}\right)
$$

if and only if $\mathcal{D}_{S R B}\left(\frac{d A}{d z}\right)=0$. By Proposition $3 \frac{d A}{d z}$ is a coboundary that is $A$ satisfies (3).

\section{Appendix A. Non-Mixing CASE.}

Observe that Anosov times rotation could satisfy the conclusions of Theorem 1 even though it is not mixing. In this appendix we give an extension of Theorem 1 to a non-mixing case. In order to explain our result let us recall some background. Given $\tau$, let $\bar{N}=M \times G$ and consider the principal extension $\bar{F}: \bar{N} \rightarrow \bar{N}$ given by $\bar{F}(x, g)=$ $(f(x), \tau(x) g)$. Recall the definition of Brin groups $[1,2]$. Given a partially hyperbolic diffeomorphism we call a sequence $P=\left\{p_{1}, p_{2} \ldots p_{n}\right\}$ a e-chain (respectively t-chain) if $p_{j+1} \in W^{u}\left(p_{j}\right) \cup W^{s}\left(p_{j}\right)$ (respectively $p_{j+1} \in W^{u}\left(p_{j}\right) \bigcup W^{s}\left(p_{j}\right) \bigcup \operatorname{Orb}\left(p_{j}\right)$. Fix a reference point $x \in M$. Given any chain $P \subset M$ with $x_{n}=x_{1}=x$ and any $g_{1} \in G$ there is unique chain $\bar{P} \subset \bar{N}$ starting at $\left(x, g_{1}\right)$ and covering $P$. $\bar{P}$ is not closed, rather $g_{n}=g(P) g_{1}$. Let $\Gamma_{t}(x)\left(\Gamma_{e}(x)\right)$ denote the set of all $g(P)$ for all closed t-chains (respectively e-chains) starting at $x$.

Proposition 7. (Brin) $([1,2])$ (a) $\Gamma_{*}(x)$ are groups. $\Gamma_{*}$ of different points are conjugated, $\Gamma_{t}$ is a normal subgroup of $\Gamma_{e}, \Gamma_{e} / \Gamma_{t}$ is cyclic. In particular $\bar{\Gamma}_{e} / \bar{\Gamma}_{t}$ is abelian.

(b) $\left(F, \nu_{\phi}\right)$ is ergodic $\Leftrightarrow \bar{\Gamma}_{e}$ acts transitively on $Y$.

$\left(F, \nu_{\phi}\right)$ is mixing $\Leftrightarrow \bar{\Gamma}_{t}$ acts transitively on $Y$.

A quantitative version of this result was obtained in [4]. Call a set $S \subset G$ Diophantine on $Y$ if there are constants $K, \sigma$ such that for any function $h$ on $Y$ with $\Delta h=\lambda h$ then there is $s \in S$ such that

$$
\|h-h \circ s\| \leq \frac{K}{\lambda^{\sigma}}\|h\|_{L^{2}} .
$$

Let $\Gamma_{t}(x, R)\left(\Gamma_{e}(x, R)\right)$ denote the set of $g(P)$ for all chains $P=$ $\left(x_{1}, x_{2} \ldots x_{n}\right)$ with $x_{1}=x_{n}=x, n \leq R, d_{W^{*}}\left(x_{j}, x_{j+1}\right) \leq R$ (if $x_{j+1}=f^{m} x_{j}$ we require that $\left.|m| \leq R\right)$. 
Proposition 8. ([4] (a) $S$ is Diophantine on $Y$ iff $S$ is Diophantine on $Y /[G, G]$ and $Y / \operatorname{Center}(G)$.

(b) $S$ is Diophantine on $Y / \operatorname{Center}(G) \Leftrightarrow$ there are no $S$-invariant functions $\Leftrightarrow S$ contains a finite Diophantine subset.

(c) $F$ is rapidly mixing $\Leftrightarrow \Gamma_{t}(R)$ is Diophantine for large $R$.

It is proven in [1] that there is an open and dense subset of pairs $(f, \tau)$ such that $\Gamma_{t}(R)=G$ for large $R$. The goal of this appendix is to prove the following statement.

Theorem 3. Suppose that $F$ is ergodic. If $\Gamma_{e}(R)$ is Diophantine for large $R$ then any solution to (1) satisfies the tame estimates (2).

Remark. I believe that the above condition is also necessary for (2) but the approach of subsection 4.4 (cf. also [4], subsection 4.3) shows only that if $\Gamma_{e}(R)$ is not Diophantine for large $R$ and $A=B-B \circ F$ then the norm of $\partial_{y}^{\alpha} B$ can not be bounded by norms of $\partial_{y}^{\beta} A$. It does not rule out the possibility that it can be bounded by norms of $\partial_{y}^{\beta_{1}} \partial_{x}^{\beta_{2}} A$, even though this seems unlikely.

Proof. Observe that the only place where we have used rapid mixing (i.e. Diophantineness of $\Gamma_{t}(R)$ ) was $(10)$. Hence we need to show that (10) holds under a weaker condition that $\Gamma_{e}(R)$ is Diophantine. To this end we estimate $\left(1-\mathcal{L}_{\lambda}\right)^{-1}$ using the series

$$
\left(1-\mathcal{L}_{\lambda}\right)^{-1}=\frac{1}{2}\left(1-\frac{1+\mathcal{L}_{\lambda}}{2}\right)^{-1}=\frac{1}{2} \sum_{j=0}^{\infty}\left(\frac{1+\mathcal{L}_{\lambda}}{2}\right)^{j} .
$$

Thus instead of Proposition 1 we need to show that there exist $C, s$ such that

$$
\left\|\left(\frac{1+\mathcal{L}_{\lambda}}{2}\right)^{n}\right\| \leq C \lambda^{s}\left(1-\frac{1}{C \lambda^{s}}\right)^{n}
$$

The proof of (14) is similar to the proof of (7) which is Proposition 4.4 of [4]. Let us describe the modifications needed. Repeating the arguments on page 184 of [4] we find that if (14) fails then for each $C_{1}, \beta_{4}$ there exist $\lambda, H$ such that $\|H\|_{C^{0}} \leq 1, L(H) \leq$ Const $\lambda$ and $\left\|\left(\frac{1+\mathcal{L}_{\lambda}}{2}\right)^{m(\lambda)} H\right\| \geq 1-|\lambda|^{-\beta_{4}}$ where $m(\lambda)=C_{1} \ln \lambda$ and $L(H)$ denotes the Lipschitz norm $H: \Sigma^{+} \rightarrow L^{2}(Y)$. As in [4] this implies that $\forall \tilde{\omega}, \hat{\omega}$

$$
\left\|\pi_{\lambda}\left(\tau_{m}(\tilde{\omega})\right) H(\tilde{\omega})-\pi_{\lambda}\left(\tau_{m}(\hat{\omega})\right) H(\hat{\omega})\right\| \leq \lambda^{-\beta_{5}}
$$

where $\beta_{5} \rightarrow \infty$ as $\beta_{4} \rightarrow \infty$. However in the present setting we also have that for all $\omega$

$$
\left\|\pi_{\lambda}(\tau(\omega)) H(\omega)-H(\sigma \omega)\right\| \leq \lambda^{-\beta_{5}}
$$


Indeed the expression for $\left[\left(\frac{1+\mathcal{L}_{\lambda}}{2}\right)^{m} H\right](\sigma \omega)$ contains among various terms

$$
(1 / 2)^{m}\left[H(\sigma \omega)+e^{\phi(\omega)} \pi_{\lambda}(\tau(\omega)) H(\omega)\right] .
$$

These two vectors should be almost collinear in the sense of [4], page 185 proving (15).

(15) implies that in our setting Lemma 4.7 of [4] holds for e-chains and not only for t-chains as in [4]. Continuing as in [4], page 186 we show that if (14) fails then this contradicts to Diophantiness of $\Gamma_{e}$. Thus (14) holds. Thus Theorem 1 holds under the assumption that $\Gamma_{e}(R)$ is Diophantine as claimed.

\section{REFERENCES}

[1] Brin M. Topological transitivity of one class of dynamical systems and flows of frames on manifolds of negative curvature, Func. An. \& Appl. 9 (1975) 8-16.

[2] Brin M. The topology of group extensions of C-systems, Mat. Zametki 18 (1975) 453-465.

[3] de la Llave R., Marco J. M. \& Moriyon R. Canonical perturbation theory of Anosov systems and regularity results for Livsic cohomology equation, Ann. Math. 123 (1986) 537-611.

[4] Dolgopyat D. On mixing properties of compact group extensions of hyperbolic systems, Israel J. Math. 130 (2002) 157-205.

[5] Dolgopyat D. On differentiability of SRB states for partially hyperbolc systems, to appear in Inv. Math.

[6] Field M., Melbourne I. \& Torok A. Decay of correlations, central limit theorem and approximation by Brownian motion for compact group extensions, Erg. Th. \& Dyn. Sys. 23 (2003) 87-110.

[7] Forni G. Solutions of the cohomological equation for area-preserving flows on compact surfaces of higher genus, Ann. of Math. 146 (1997) 295-344.

[8] Gorodetskii A. S. \& Ilyashenko Yu. S. Some properties of skew products over a horseshoe and a solenoid, Proc. Steklov Inst. Math. 231 (2000) 90-112.

[9] Hurder S. \& Katok A. Differentiability, rigidity and Godbillon-Vey classes for Anosov flows, Publ. IHES 72 (1990) 5-61.

[10] Journe J.-L. A regularity lemma for functions of several variables, Rev. Mat. Iberoamericana 4 (1988) 187-193.

[11] Katok A.\& Kononenko A. Cocycles' stability for partially hyperbolic systems, Math. Res. Lett. 3 (1996) 191-210.

[12] Katok A. \& Spatzier R. J. First cohomology of Anosov actions of higher rank abelian groups and applications to rigidity, Publ. IHES 79 (1994) 131-156.

[13] Parry W. \& Pollicott M. Zeta Functions and Periodic Orbit Structure of Hyperbolic Dynamics, Asterisque v. 187-188 (1990).

[14] Shub M. \& Wilkinson A. Pathological foliations and removable zero exponents, Inv. Math. 139 (2000) 495-508.

[15] Veech W. Periodic points and invariant pseudomeasures for toral endomorphisms, Erg. Th. \& Dyn. Sys. 6 (1986) 449-473. 